\section{Biodegradation of DDT}

\section{Ashish Chauhan ${ }^{1 *}$ and Jashwant Singh ${ }^{2}$}

${ }^{1}$ National Institute of Pharmaceutical Education and Research, Mohali, Punjab, India ${ }^{2}$ Indian Institute of Technology, Kanpur, India

D.D.T. (1,1,1-trichloro-2,2-bis (p-chlorophenyl) ethane) is one of the best synthetic pesticides. First synthesized in 1874, its properties were not discovered until 1939. It was used to control mosquitoes spreading malaria, typhus and other insect borne diseases among both military and civilian populations. After the war DDT was made available for use as an agricultural insecticide. It is an organo-chlorine insecticide highly hydrophobic, colorless, crystalline solid with a weak, chemical odor. It is nearly insoluble in water but has a good solubility in most organic solvents, fats and oils. DDT does not occur naturally, but is produced by the reaction of chloral $\left(\mathrm{CCl}_{3} \mathrm{CHO}\right)$ with chloro benzene $\left(\mathrm{C}_{6} \mathrm{H}_{5} \mathrm{Cl}\right)$ in the presence of sulphuric acid, which acts as a catalyst. Commercial DDT is a mixture of several related compounds. It has significant amount of (15\%). Dichloro-diphenyl-dichloro-ethylene (DDE) and dichloro-diphenyl-dichloroethane (DDD).

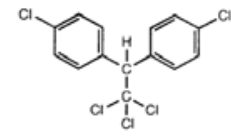

4,4-DDT

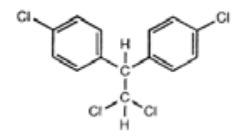

$4,4^{4}-\mathrm{DDD}$

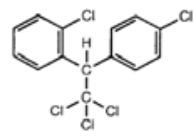

2,4'-DDT

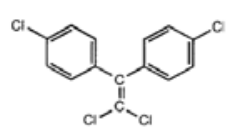

4,4-DDE
Since DDT residue are liphophilic they tend to accumulate in the fatty tissues of the ingesting organism along the food chain. Almost all the food stuffs including processed foods have been shown to contain high level of DDT residues. High level of DDT and its metabolites have been detected a human adipose tissues, blood plasma, liver, brain, placenta and even in breast milk. It is reported to be a potential endocrine disruptor in both avian and mammals. Resulting egg shell thinning, impaired male reproductive ability, interference with sex hormones, causes cancer and many other nervous diseases.

Biodegradation is the process by which organic substances are broken down by the enzymes produced by living organisms. The term is often used in relation to ecology, waste management and environmental remediation called bioremediation. Organic material can be degraded aerobically, with oxygen or an aerobically, without oxygen. A term related to biodegradation is bio mineralization, in which organic matter is converted into minerals. Some microorganism have the astonishing, naturally occurring, microbial catabolic diversity to degrade or transform hydrocarbons (e.g. oil), polychlorinated biphenyls (PCBs), and poly aromatic hydrocarbons (PAHS).

Some microorganisms that are capable to degrade DDT are:

\section{Bacteria:}

Escherichia coli

Enterobacter aerogens.

Enterobacter cloacae.

Klebsiella pneumonia.

\section{Pseudomonas aeruginosa.}

Pseudomonas putida.

Bacillus species.

Hydrogenomonas.

Fungi:

Saccharomyces cervisiae.

Phanerochaete chrysosporium.

Trichoderma viridae.

Although DDT is metabolized through co-metabolism pathway by a great number of facultative and obligate microorganism under suitable conditions. No microorganism is known that utilizes DDT as the only source of carbon and energy. DDT and some of its transformations product specially the enentiomers of $o$ ' $p$ ' isomers are shown to be estrogenically active. DDD and DDE are metabolized product of DDT that are produced by microbial degradation, chemically or by photochemical reactions. DDD (1, 1-dichloro-2, 2-bis (p-chlorophenyl) ethane) is formed by DDT through reductive dechlorination either microbial mediated way or as chemical reaction and dehalogenation of DDD to produce DDM (4,4-di chloro-diphenyl methane) under subsequent oxidative conditions hydroxylation and ring cleavage were observed. DDE is formed by DDT through photochemical reaction in presence of sunlight and through dehydro chlorination in bacteria. These products are metabolized very slowly.

There are various method for biological degradation of DDT like fugal degradation, aerobic degradation and anaerobic degradation. Comparison to Fugal degradation, aerobic degradation and anaerobic degradation has various advantage such as economical, easy, simple and needs no extra efforts. Chemical representation of DDT degradation by aerobic and anaerobic method is as follows:

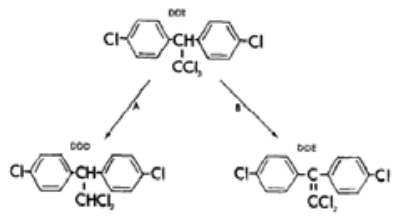

De chlorination of DDT A-Anaerobic de chlorination; B-aerobic dehydro chlorination

*Corresponding author: Ashish Chauhan, National Institute of Pharmaceutical Education and Research, Mohali, Punjab, India, Tel: 9464616773; E-mail: aashishchauhan26@gmail.com

Received January 30, 2015; Accepted February 03, 2015; Published February 10, 2015

Citation: Chauhan A, Singh J (2015) Biodegradation of DDT. J Textile Sci Eng 5 183. doi: $10.4172 / 2165-8064.1000183$

Copyright: (c) 2015 Chauhan A, et al. This is an open-access article distributed under the terms of the Creative Commons Attribution License, which permits unrestricted use, distribution, and reproduction in any medium, provided the original author and source are credited. 